Disponível em:

http://editora.unoesc.edu.br/index.php/race

Race, Joaçaba, v. 14, n. 3, p. 1119-1144, set./dez. 2015

\title{
PROGRAMA DE APRENDIZAGEM: IMAGEM E SATISFAÇÃO NA VISÃO DOS APRENDIZES
}

\section{Learning program: image and satisfaction in the view of learners}

\begin{abstract}
Suzete Antonieta Lizote
E-mail:emaildasuzy@gmail.com

Doutora em Administração e Turismo pela Universidade do Vale do Itajai; Mestre em Contabilidade e Controladoria pela Fundação Universidade Regional de Blumenau; Professora do Curso de Ciências Contábeis da Universidade do Vale do Itajaí. Endereço para contato: Rua Uruguai, 458, Centro, 88302-202, Itajai, Santa Catarina, Brasil.
\end{abstract}

\section{Miguel Angel Verdinelli} E-mail:nupad@univali.br Doutor em Ciências pela Universidade de São Paulo; pós-doutorado em Ciências Sociais pela Universidad de Alicante, Espanha; Professor do Mestrado e Doutorado em Administração na Universidade do Vale do Itajaí.

\section{Elisa Couto Laurentino}

E-mail: elisacoutolaurentino@hotmail.com Pós-graduanda em Gestão tributária e finanças na Universidade do Vale do Itajaí.

\section{Luana da Silva Santos}

E-mail:1uana@mosarte.com.br

Pós-graduanda em Gestão tributária e finanças na Universidade do Vale do Itajaí.

Thais Cristina Melim Petrelli

E-mail: thais.melimpetrelli@hotmail.com

Pós-graduanda em Gestão tributária e finanças na Universidade do Vale do Itajaí.

Artigo recebido em 12 de fevereiro de 2015. Aceito em 30 de junho de 2015. 


\section{Resumo}

A formação técnico-profissional de jovens é importante para sua inserção no mercado de trabalho. A associação da experiência profissional com o estudo representa para muitos deles o caminho para a melhoria das condições de vida. Diante desse contexto, nesta pesquisa se analisou a imagem e a satisfação dos aprendizes sobre o programa de aprendizagem do Instituto Crescer. Para estabelecer o marco conceitual, foi consultada a legislação que trata desse tema no Brasil. Este estudo de abordagem quantitativa, por sua natureza, classificase como aplicado e segundo seu objetivo descritivo. A população estava composta por duas turmas de aprendizes: os que ingressaram recentemente ao curso e possuem aulas diárias e os que já ingressaram no mercado de trabalho e participam de palestras quinzenais. Os dados foram obtidos por meio de um questionário de autopreenchimento, no qual se levantaram as informações pessoais e as expectativas a respeito do trabalho. A percepção dos aprendizes sobre a imagem e a satisfação com o programa era colhida em um segundo bloco, que estava dividido em três categorias: ensino, instituição promotora e desenvolvimento pessoal. Os resultados apontaram que em todas as categorias as médias foram mais do que $80 \%$ do valor máximo possível tanto para a imagem quanto para a satisfação, seja para os aprendizes iniciantes seja para os mais antigos. Os resultados também confirmam diferenças entre as percepções dos grupos. Os valores mais elevados, quando ocorreram, foram para os estudantes que entraram recentemente, para os menores de 16 anos e para as mulheres. Conclui-se que as respostas dos aprendizes são muito favoráveis ao desenvolvimento do programa.

Palavras-chave: Aprendizes. Programa de aprendizagem. Imagem. Satisfação.

\section{Learning program: image and satisfaction in the view of learners}

\section{Abstract}

Technical and professional training of young people is important for their integration into the labor market. For many of them, the association of professional experience to the study means the way for the improvement of living conditions. Given this context, in this study we analyzed the image and satisfaction of learners about Instituto Crescer's learning program. To establish the conceptual framework, the Brazilian legislation addressing this issue was consulted. This study of quantitative approach, by its nature, is classified as applied and according to its descriptive goal. The population was composed of two groups of learners: those who recently joined the program and have daily classes and those who have entered the labor market and participate in biweekly lectures. The data of were collected through a self-report questionnaire. In addition to raising the personal information and expectations regarding work, the questionnaire had a block that collected data about the image and satisfaction with the program. This block was divided into three categories: education, promoting institution and personal development. The results showed that in all three categories, the values of means were more than $80 \%$ of the maximum possible value for both the image and to the satisfaction. The results also confirm differences between the perceptions of the groups. The higher values, when they occurred, were for students who entered recently, for the ones under 16 years old and for women. We conclude that the learners' answers are very favorable to the development of the program. Keywords: Learners. Learning program. Image. Satisfaction. 


\section{INTRODUÇÃO}

O trabalho, durante toda a história da sociedade capitalista, tem sido um meio necessário para o homem promover o autosustento e, muitas vezes, amparar financeiramente uma família inteira. Nesse contexto, o jovem, diante de diversos determinantes, como baixos salários pagos aos seus pais, vê-se obrigado a entrar no mercado de trabalho precocemente, por necessidade de sobrevivência (KASSOUF; MCKEE; MOSSIALOS, 2001; BROWN; STERN; DEARDOFF, 2002).

$\mathrm{O}$ ideal seria que o jovem não trabalhasse, pois, às vezes, o labor pode prejudicar a aquisição de conhecimentos e o desenvolvimento de habilidades pertinentes a esse período de formação do adulto. A solução encontrada para essa adversidade foi a criação de um contrato de aprendizagem, com o qual o aprendiz é inserido no mercado de trabalho de maneira a preservar o tempo reservado para o estudo e ainda conciliar o trabalho com uma formação técnica.

Os programas de qualificações começaram a ser reformulados somente no final dos anos 1990, principalmente por meio dos Programas de Geração de Empregos e Formação Profissional, quando novas visões acerca dos jovens foram surgindo, considerando-os protagonistas e responsáveis pelo futuro (FREITAS; PAPPA, 2008; CRISPIM; GODOY, 2010). Considera-se protagonismo juvenil o processo no qual o jovem deve participar como ator principal, desenvolvendo-se como pessoa, ou aprendendo a ser, e capacitando-se para o trabalho, ou aprendendo a fazer (OLIVEIRA, 2009).

A ponte do conhecimento necessário para desempenhar as funções do contrato de aprendizagem vem das escolas de aprendizagem ou dos serviços sociais de aprendizagem, nos quais os jovens são matriculados por conta própria ou pela empresa contratante. Nesse local, o jovem recebe instruções básicas necessárias para que realize o serviço ao que é contratado, complementando o aprendizado da escola regular em seu contraturno.

De acordo com o Manual da Aprendizagem (MINISTERIO DO TRABALHGO E EMPREGO, 2009), a aprendizagem no Brasil, que estava regulamentada na Consolidação das Leis do Trabalho (BRASIL, 1943) no capítulo referente ao trabalho do menor, passou por um processo de modernização por meio da Lei n. 10.097/00 (BRASIL, 2000) e da Lei n. 11.180/05 (BRASIL, 2005b). Esta última e o Decreto n. 5.598 (BRASIL, 2005a), que regulamenta a contratação de aprendizes, definem as condições que se devem cumprir. Entre elas, cabe destacar a extensão da faixa etária, que passou dos 18 para os 24 anos, a definição da cota 
conforme a organização e a exclusão, para seu cálculo, do número de funcionários cujo labor demanda habilitação de nível superior ou técnico e dos cargos diretivos e de confiança. Assim, pode ser aprendiz o adolescente ou o jovem com idade entre 14 e 24 anos, que esteja matriculado e frequentando a escola, caso não tenha concluído o ensino médio, devendo estar inscrito em um programa de aprendizagem.

O processo de aprendizagem visa preparar o jovem para desempenhar atividades profissionais e ter capacidade de discernimento para lidar com diferentes situações no universo corporativo. Nesse sentido, nesta pesquisa buscou-se a resposta ao seguinte questionamento: Qual a imagem e a satisfação que têm os aprendizes atendidos pelo Instituto Crescer da Cidade de Itajaí, Santa Catarina, a respeito do programa de formação para o trabalho?

No intuito de responder ao questionamento proposto, o objetivo geral com este estudo foi analisar a imagem e a satisfação dos aprendizes do Instituto Crescer sobre o programa de aprendizagem. Para seu alcance, definiu-se o seguinte objetivo específico: investigar a percepção de imagem e a satisfação dos aprendizes em relação aos aspectos do ensino, da instituição promotora e do desenvolvimento pessoal.

Sobre a dificuldade do jovem em seu processo de inserção no mercado de trabalho, Câmara e Sarriera (2001) relatam a existência de múltiplos fatores, entre eles: a qualidade da educação, muitas vezes, não adequada às novas exigências de mercado, a falta de experiência profissional do jovem e as dificuldades econômicas de grande parte das famílias brasileiras. Nesse sentido, oferecer para o aprendiz o direito ao início de uma formação profissional, assegurando desenvolvimento intelectual, atribui para a educação um papel essencial, visto que os processos educacionais, escolares ou não, constituem-se em práticas sociais mediadoras e formadoras da sociedade.

Ao estudar a relação entre o capital humano e o trabalho dos menores, Fan (2004) desenvolveu um modelo que, ao incluir na função de formação do capital o tempo e o valor do salário, verifica-se que um aumento na produtividade pode levar a um aumento tanto do trabalho quanto do capital humano. Portanto, um pequeno aumento do trabalho não vai, necessariamente, afetar de maneira negativa o capital humano dos jovens, pois o impacto positivo do aumento dos recursos financeiros na educação pode ser maior do que o impacto da redução do tempo de estudo.

Com base nesses argumentos, salienta-se a relevância desta pesquisa ao destacar que quando os jovens deixam de estudar, em razão da necessidade de trabalhar, têm maiores possibilidades de se tornarem adultos com baixa renda e, assim, criar um círculo vicioso ao ter que mandar seus filhos trabalhar (EMERSON; 
SOUZA, 2003). Mas, quando o jovem se torna aprendiz, mantendo seus estudos, passa a assumir compromissos e um novo papel na sociedade, pois, por ter oportunidades, poderá decidir por uma profissão de seu interesse e compatível com suas aptidões. Percebe-se, desse modo, que o desenvolvimento pessoal e do capital humano tem relação direta com a escolha vocacional, em conjunto aos interesses e habilidades do aprendiz.

Por outro lado, é preciso estudar e elaborar as políticas públicas da juventude de maneira integrada com outros setores da sociedade, uma vez que o trabalho dos jovens é bastante comum em muitos países, tendo causas diversas; geralmente se considera a pobreza como a principal razão, contudo, estudos teóricos e empíricos demostram que há outros fatores, entre eles o acesso ao crédito, às oportunidades do mercado de trabalho e a qualidade da escola (ERSADO, 2003). Ou seja, as ações devem ser divididas entre o poder do governo, das entidades sem fins lucrativos e das empresas privadas. Entre aquelas, destaca-se a oferta de educação com qualidade, a qual, aliada ao aproveitamento dos jovens nas escolas de aprendizagem, possibilita a esse segmento da população o desenvolvimento do capital humano, favorecendo, assim, sua empregabilidade futura.

Após esta introdução, a estrutura do artigo compreende o aporte teórico necessário à compreensão dos itens a serem estudados, quais sejam: aprendiz, contrato de aprendizagem, satisfação e imagem. Na seção seguinte, relacionam-se os procedimentos metodológicos, seguidos da descrição e análise dos dados, em que se mostram os resultados obtidos. Por último, são apresentadas as considerações finais da pesquisa e disponibilizadas as referências.

\section{MARCO TEÓRICO}

\subsection{APRENDIZ}

Aprendiz é o jovem com idade entre 14 e 24 anos, que esteja cursando a escola regular, até a conclusão do ensino médio, e esteja inscrito em um curso de aprendizagem (art. 428, caput e $\mathbb{\$} 1^{\circ}$, da Consolidação das Leis do Trabalho - CLT). Entre estes, adolescentes entre 14 e 18 anos possuem a prioridade no momento da contratação para o cargo de aprendiz.

A inserção desses jovens em cursos de aprendizagem, segundo Frigotto (2010), influencia-os a explorarem seus interesses por meio da educação, envolvendo- 
se em atividades que contribuem para solucionar problemas existentes e que ultrapassem os limites preestabelecidos pelo ambiente em que ele está inserido. De modo geral, o adolescente procura o primeiro emprego por necessidade, para prover a si e contribuir com o orçamento doméstico, logo, a realidade de empregar-se como jovem aprendiz lhe oportuniza suprir essa necessidade de forma lícita, evitando que este se perca nas drogas ou entre para o crime (MINISTÉRIO DO TRABALHO E EMPREGO, 2009).

Antes da Lei n. 10.097/2000, que altera os dispositivos da Consolidação das Leis do Trabalho à contratação de aprendizes, as empresas contratavam esses jovens para a realização de pequenos trabalhos. Raramente existia vínculo empregatício reconhecido e a contratação era feita de forma precária, não havendo assinatura na carteira de trabalho do jovem. Para Santos (2003, p. 18),

\begin{abstract}
O adolescente não se qualificava para ofício algum, dada a simplicidade das tarefas que executava. Tinha poucos direitos de natureza trabalhista e nenhum previdenciário. Para trabalhar, deixava de estudar ou tinha prejudicado o aproveitamento escolar, o que comprometia sua manutenção no mercado de trabalho. Aos 18 anos de idade, surpreendia-se desempregado, desqualificado e desamparado. Além disso, se fosse perspicaz, perceberia que fora explorado: prestava serviços como qualquer empregado (talvez até tomando o posto de trabalho de um pai de família) e não usufruíra os direitos correspondentes. Havia sido objeto do oportunismo de algum empresário ávido de lucro.
\end{abstract}

Com o advento da referida Lei, a inserção desses jovens como aprendizes no mercado de trabalho, conforme enfatiza Ferraz (2006), influencia na sua maturação, adquirindo responsabilidade de forma a integrá-los com a sociedade, capacitandoos na vida profissional e concedendo-lhes um diferencial entre a população de trabalhadores desempregados ou desqualificados. Porém, essa inserção, segundo Macedo (2006), não se trata da possibilidade de as empresas alcançarem seu objetivo econômico por meio de uma mão de obra barata; segundo ele, os benefícios na qualificação dos jovens também têm impacto no desenvolvimento econômico do País, uma vez que formam trabalhadores melhores e mais competitivos, influenciando, assim, na qualidade dos produtos nacionais.

Nessa linha de pensamento, Josviak (2009) coloca que a ideia de proporcionar ao aprendiz o direito ao início de uma formação profissional, garantindo desenvolvimento intelectual, atribui à educação um papel fundamental, 
visto que os processos educacionais, escolares ou não, traduzem-se em práticas sociais mediadoras e formadoras da sociedade.

\subsection{CONTRATO DE APRENDIZAGEM}

Publicada no ano 2000, a Lei n. 10.097 estabelece as regras aplicáveis para a contratação de jovens aprendizes. Com ela, houve a alteração também da menoridade do trabalhador de 12 para 14 anos, também foi definindo o limite para contratação como aprendiz, passando de 16 para 24 anos, e tornou-se obrigatório que os empregadores de qualquer natureza, além de contratar, matricularem o jovem em cursos dos Serviços Nacionais de Aprendizagem ou, na hipótese de esta não possuir vagas, em outras entidades qualificadas em formação técnico-profissional.

A Lei supracitada também passou a obrigar os estabelecimentos de qualquer natureza (excluindo-se as microempresas e empresas de pequeno porte e as entidades sem fins lucrativos) a empregarem e matricularem nos cursos e instituições formadoras um número de aprendizes equivalente a 5\%, no mínimo, e 15\%, no máximo, dos trabalhadores existentes em cada estabelecimento, cujas funções demandem formação profissional.

A Instrução Normativa SIT n. 26 (BRASIL, 2001) dispõe:

Para a definição das funções que demandam formação profissional deverão ser considerados a Classificação Brasileira de Ocupações (CBO) e os seguintes fatores:

I - o nível das capacidades profissionais e dos conhecimentos técnico-teóricos requeridos para o exercício da atividade profissional;

II - a duração do período de formação necessário para a aquisição das competências e habilidades requeridas; e,

III - a adequação da função às necessidades da dinâmica de um mercado de trabalho em constante mutação.

Nesse sentido, argumenta Soares (2007, p. 17),

Há uma grande dificuldade por parte das empresas da área de comercio de bens e serviços de entender a forma de aplicação da Lei n. 10.097/00, que determina que elas devam cumprir um sistema de cotas para a contratação de menores aprendizes, pois, para o setor industrial, essa matéria já é corriqueira há décadas. 
Com a lei da aprendizagem, veio a obrigatoriedade na contratação de forma lícita, isto é, com a assinatura na carteira de trabalho do jovem, por meio de um contrato de aprendizagem. Com base no Manual da Aprendizagem (MINISTÉRIO DO TRABALHO E EMPREGO, 2009), formulado pela Secretaria de Políticas Públicas de Emprego do Ministério do Trabalho e Emprego, o contrato de aprendizagem é ajustado por escrito e por prazo determinado, em que o empregador se compromete a assegurar ao aprendiz inscrito em programas de aprendizagem, formação técnico-profissional metódica, compatível com seu desenvolvimento físico, moral e psicológico. E o aprendiz, por sua vez, compromete-se a executar, com zelo e diligência, as tarefas necessárias a essa formação.

Assim, conclui-se que a introdução do jovem no mercado de trabalho passa a ocorrer da forma menos prejudicial possível, obtendo a oportunidade de receber um salário pelo serviço prestado, com o apoio de todos os direitos trabalhistas e previdenciários, aproximando-o sempre dos estudos, pois outra exigência é que o jovem deve frequentar, ao longo do contrato, escola regular e ensino técnico profissionalizante.

\subsection{SATISFAÇÃO E IMAGEM}

A concorrência, acirrada ou não, estimula as organizações a inovarem seus produtos e serviços, tendo como foco o atendimento às necessidades do consumidor. Conforme colocava McKenna (1992), para a empresa, o decisivo é o que os clientes pensam a seu respeito. Isso faz com que a organização desenvolva um trabalho que atenda às expectativas de sua clientela, e, portanto, é imperativo conhecer as verdadeiras necessidades e desejos dela.

Dessa forma, são necessárias algumas medidas para avaliar as satisfações dos clientes. Segundo Kotler (1998), a satisfação é o sentimento de desapontamento ou prazer resultante da comparação do desempenho esperado de um produto ou serviço em relação às expectativas da pessoa. De Toni (2005) assinala que um bom programa de estudo da satisfação permite monitorar o desempenho da empresa em relação aos seus concorrentes, compreender os aspectos de maior impacto na percepção dos consumidores, analisar a sintonia entre a direção da empresa e a expectativa dos seus clientes, entre outros argumentos. Para Churchill e Peter (2003), ao desenvolver um processo de avaliação da satisfação do cliente, as empresas obtêm um panorama 
da opinião deles em relação ao desempenho, indicando caminhos para as decisões futuras de aperfeiçoamento nas diretrizes organizacionais.

Neves e Ramos (2002), por sua vez, argumentam que a satisfação tem um efeito direto e significante na imagem, ou seja, o alto nível de satisfação do consumidor lida com uma imagem positiva por parte do cliente. $\mathrm{O}$ conceito de imagem, conforme destacam Schuler e De Toni (2004), envolve emoções, percepções, impressões, suposições e também expectativas. Sampaio (1999, p. 248) já argumentava que "[...] a imagem é o conceito que as pessoas têm ou formam sobre as coisas e querendo ou não, gostem ou não, tudo e todos passam uma imagem, inclusive a respeito de si próprios."

Na concepção de De Toni, Milan e Schuler (2005), a imagem é capaz de influenciar e direcionar o comportamento das pessoas, pois se constitui em um dos componentes intelectuais mais importantes do ser humano na vida em sociedade. Dessa forma, pode-se inferir que o processo de construção de imagem de uma empresa envolve a relação direta do indivíduo com os seus produtos ou serviços, informações transmitidas pela organização por meio do seu processo de comunicação, os contatos com os membros da organização, a influência de opiniões de terceiros, entre outros.

\section{METODOLOGIA}

\subsection{CLASSIFICAÇÃO DA PESQUISA}

Existem diversas classificações para a pesquisa científica, sendo uma das formas básicas fazê-la conforme seja finalidade. Segundo propõem Cervo e Bervian (2003), por sua natureza, pode-se classificar como pura ou aplicada. No primeiro tipo, o pesquisador tem como propósito o próprio conhecimento, enquanto na pesquisa aplicada buscam-se respostas ou soluções a questionamentos concretos, com a finalidade de contribuir para fins práticos. Sob esse enfoque, a presente pesquisa classifica-se como aplicada, pois visa responder a uma questão que permeia a realidade dos programas destinados à formação para o trabalho dos aprendizes.

Por outra parte, Andrade (1999) classifica a investigação científica considerando o objetivo do estudo e a distingue em exploratória, descritiva e explicativa. A pesquisa exploratória realiza-se quando se objetiva examinar algo com poucos estudos prévios, procurando informações iniciais sobre determinado 
assunto. Já o estudo descritivo pretende responder às perguntas de pesquisa a partir das características que apresentam os fenômenos, estimando, por exemplo, proporções em uma população na qual ele se manifesta. Também se busca descobrir a existência de associações entre diferentes variáveis que estão sendo consideradas. Finalmente, a pesquisa explicativa, conforme salienta a autora, é mais complexa e implica registrar, analisar, interpretar e tipificar o que está sendo estudado com o intuito de identificar os fatores que determinam a ocorrência das relações, seu sentido, sua intensidade, entre outras características.

O tipo de pesquisa utilizado para a elaboração do artigo foi a forma descritiva, pois busca conhecer situações e suas relações sem a interferência do pesquisador. Cervo e Bervian (2003, p. 66) lecionam que "A pesquisa descritiva observa, registra, analisa e correlaciona fatos ou fenômenos (variáveis) sem manipulá-los.”

Pelos métodos de análise, a abordagem é quantitativa, pois se levantaram dados que possibilitaram mensurar variáveis para testar hipóteses e generalizar resultados, o que corresponde a uma avaliação quantitativa, conforme Martins e Theóphilo (2009) implica organizar, sumarizar, caracterizar e interpretar os dados numéricos coletados.

\subsection{POPULAÇÃO E DADOS OBTIDOS}

A população foi composta por duas turmas de aprendizes, uma delas compreende os que ingressaram recentemente ao curso e possuem aulas diárias, totalizando 60 jovens. A outra turma é composta pelos aprendizes que já estão inseridos no mercado de trabalho e frequentam as aulas do programa quinzenalmente. Estão incluídos nesse grupo 45 jovens.

Embora se tenha procurado realizar um censo, os dados obtidos corresponderam a 56 respondentes da turma de ingressantes ao programa e a 35 aprendizes que já estavam trabalhando. Esses números constituíram a população acessível, pois todos os aprendizes colaboraram respondendo ao instrumento de pesquisa empregado. Isto é, os dados da presente pesquisa de survey (BABBIE, 2003) foram obtidos por meio de um questionário de autopreenchimento aplicado aos jovens aprendizes atendidos pelo Instituto Crescer enquanto eles participavam das aulas. 


\subsection{INSTRUMENTO E PROCEDIMENTOS}

$\mathrm{O}$ instrumento de pesquisa aplicado possui três blocos. No primeiro recolhem-se as informações do respondente, o segundo refere-se às expectativas em relação à aprendizagem, à empresa onde o aprendiz poderia trabalhar e ao mercado de trabalho em geral e, finalmente, o último bloco contempla os aspectos centrais do trabalho, isto é, a imagem e a satisfação que os aprendizes possuem do ensino, da instituição promotora da aprendizagem e de suas expectativas de desenvolvimento pessoal.

Nesse terceiro bloco, dispuseram-se 10 aspectos do programa a serem avaliados em uma escala intervalar de 10 pontos, estabelecendo uma nota para a imagem e outra para a satisfação. Considerou-se a imagem um componente emocional, que corresponde aos sentimentos de simpatia ou rejeição que algo ocasiona, e a satisfação foi entendida como uma medida de que as necessidades foram atendidas ao se considerar o que se está avaliando. As três primeiras frases referiamse ao ensino, as três seguintes, à instituição promotora da formação, e as quatro últimas, às expectativas que o aprendiz tem sobre seu desenvolvimento pessoal.

Os dados coletados foram organizados em uma planilha do Excel, na qual se fez o pré-tratamento destes. Inicialmente, sinalizaram-se os dados faltantes e constatou-se que havia 54 células com ausência de dados para o terceiro bloco, representando 3,07\% do total. Três respondentes foram excluídos, porque apresentavam mais que $50 \%$ das questões sem responder, restaram, então, 14 células não respondidas, que foram preenchidas com o valor da mediana da questão.

Em relação à primeira parte do questionário, informações pessoais e expectativas, foram feitas análises descritivas utilizando-se planilhas eletrônicas do Excel. As informações foram separadas por categorias e, posteriormente, calculadas as devidas porcentagens para a apresentação dos resultados.

Os dados levantados com o terceiro bloco foram importados ao software Statistica para se fazerem as análises da imagem e da satifação que os aprendizes manifestaram. Os aspectos considerados, ensino, instituição e expectativa de desenvolvimento pessoal, foram trabalhados a partir do valor médio das somativas das pontuações atribuídas. Desse modo, a imagem ou satisfação com o ensino e a instituição, que são mensuradas por três frases, podem atingir na soma um valor mínimo de 3, caso o aprendiz assinale 1 para todas elas, ou um máximo de 30, se a pontuação atribuída for 10. Para o terceiro aspecto, que se avalia com quatro frases, os valores mínimos e máximos podem ser 4 e 40, respectivamente. 
Os contrastes das médias foram feitos em três condições diferentes. A primeira entre os ingressantes e os que já estão inseridos no mercado de trabalho, a seguir, entre os que têm até 16 anos e os maiores dessa idade e, finalmente, entre os aprendizes de gênero feminino e masculino.

As comparações de médias para amostras independentes pelo teste $\mathrm{t}$ foram feitas após serem avaliados os pressupostos de normalidade e homogeneidade da variância. Quando o teste de Levene, que mede esse último aspecto, mostrou heterogeneidade, fez-se o cálculo do valor t com estimativas separadas de variância. Utilizou-se $5 \%$ para o nível de significância $(\alpha=0,05)$, então, caso a significância do resultado, o valor-p, seja menor que 0,05 , admite-se que ocorrem diferenças entre as médias com confiança de $95 \%$ no resultado.

\section{RESULTADOS}

\subsection{OBJETO DO ESTUDO: INSTITUTO CRESCER}

O estudo teve como universo de pesquisa a totalidade dos jovens aprendizes inscritos no Instituto Crescer-Movimento, Cidadania e Juventude, localizado no centro de Itajaí, Santa Catarina. Uma de suas atividades é o Projeto Crescer, que proporciona aos adolescentes condições de participação na sociedade como profissionais, viabilizando sua formação para a inserção no mercado de trabalho como menores aprendizes.

Esse instituto tem como missão promover o exercício pleno da cidadania, da ética e da responsabilidade, por meio da aprendizagem profissional, para a inserção de jovens e adolescentes no mercado de trabalho. Contribui, assim, para que os jovens se tornem cidadãos socialmente proativos, desenvolvendo habilidades técnicas e pessoais que resultam em maior capacidade de conseguirem seu primeiro emprego.

O programa desenvolvido no Instituto comporta dois tipos de alunos. Os jovens que ainda não estão inseridos no mercado de trabalho, aos quais proporciona um curso preparatório com duração de 10 meses, e os jovens que já estão inseridos no mercado de trabalho, disponibilizando para eles aulas quinzenais. 


\subsection{ANÁLISE DESCRITIVA}

Os questionários foram aplicados de forma individual aos alunos que estão iniciando sua formação no Instituto Crescer e aos que já estão inseridos no mercado de trabalho. Dos 91 questionários considerados válidos, 61 foram respondidos por mulheres e 30 por homens.

Das 61 mulheres respondentes, 36 estão iniciando na escola de aprendizagem e 25 já estão trabalhando. Em relação aos homens, 20 estão na categoria de iniciantes e 10 já estão trabalhando.

A respeito da idade dos jovens que responderam ao questionário, 17 possuem 15 anos de idade, 28 têm 16, com 17 anos, há 27 jovens, 14 possuem 18 anos, com 19, há quatro aprendizes e somente um completou a idade de 20 anos.

Nota-se que todos os jovens de 15 anos estão na categoria de ingressantes, na qual o aluno mais velho possui 18 anos. Entre os jovens que já estão no mercado de trabalho, o mais jovem tem 16 anos e o mais velho, 20.

Em relação às informações pessoais dos menores, constatou-se que $74,72 \%$ deles moram em casa própria, 19,78\%, em casa alugada, 1,10\%, em casa financiada e 4,40\%, em vivendas emprestadas. Acerca dos residentes de uma mesma casa, 1,10\% dos jovens declararam morar com apenas uma pessoa, 2,20\%, com duas pessoas, com três a porcentagem chega a 19,78\%, morando com quatro pessoas há $42,86 \%$ dos jovens, 20,88\% vivem com cinco pessoas, com seis, 7,69\% dos aprendizes e $5,49 \%$ moram com mais de sete moradores.

Quando questionados sobre quantas pessoas contribuem para a renda familiar, 45,05\% dos jovens responderam que são duas pessoas. A renda familiar total dos jovens varia entre um e seis ou mais salários-mínimos, porém, apenas 7,69\% dos jovens estão nessa última condição.

O grau de escolaridade dos jovens varia entre ensino fundamental incompleto, comportando 2,20\% deles, ensino fundamental completo, com 5,49\% dos jovens, ensino médio incompleto, com 72,53\%, e ensino médio completo, $19,78 \%$. Percebe-se que a maioria dos jovens possui ensino médio incompleto.

Sobre a importância dos cursos desenvolvidos, relacionados à formação para o trabalho, $76,92 \%$ dos alunos entendem ser alta, 21,98\% consideraram como média e $1,10 \%$ preferiu não responder ao questionamento. Relacionando as aulas lecionadas e a definição de uma carreira profissional, 70,33\% dos jovens afirmam que as aulas ajudam na escolha profissional, $28,57 \%$ entendem que ajudam de forma intermediária e 1,10\%, que não ajudam. Ainda acerca da escola de aprendizagem, 
os jovens esperam primordialmente adquirir novos conhecimentos e como última opção, conhecer pessoas e fazer novas amizades.

Quanto aos potenciais empregadores dos aprendizes, 63,74\% dos jovens entendem que o trabalho a ser executado pode ajudar a definir uma carreira profissional que queiram seguir, 26,37\% acreditam que essa ajuda ocorre apenas de forma intermediária, 6,59\% acreditam que não ajuda e 3,30\% não responderam. Acerca da lei de aprendizagem, $41,76 \%$ dos jovens entendem que mesmo sem a lei conseguiriam estar trabalhando com a sua idade, 52,75\% acreditam que não conseguiriam estar trabalhando sem o advento da lei e 5,49\% abstiveram-se de responder.

Dos 91 respondentes, 89 têm interesse em fazer um curso superior, ou seja, quase a totalidade dos jovens. Entre os cursos escolhidos, os que prevalecem são Direito e Psicologia, porém, 16 jovens ainda estão em dúvida em mais de um curso. A maioria dos jovens (96,70\%) acredita que ser aprendiz é importante para sua experiência profissional, de modo que, posteriormente, serão admitidos em outros empregos com mais facilidade. E, finalizando, em relação as expectativas do jovem no mercado de trabalho, 67 deles $(73,6 \%)$ esperam formar-se na universidade e exercer uma profissão.

\subsection{ANÁLISE DA IMAGEM E SATISFAÇÃO}

No que concerne à imagem e satisfação, as três frases relacionadas ao ensino abordaram os seguintes temas: as disciplinas oferecidas pelo programa, os professores que as lecionam e as instalações e o local onde se realizam os estudos. As comparações sempre foram feitas considerando-se três condições: a de iniciante no programa contra os que já estão trabalhando, os que têm até 16 anos confrontados aos maiores dessa idade e os de sexo feminino comparados aos de sexo masculino.

No Gráfico 1, exibem-se as comparações das notas médias da imagem a respeito do ensino, calculadas a partir da soma das pontuações atribuídas às três frases. No Gráfico 1A, apresenta-se a comparação dos valores médios apresentados pelo grupo de alunos que está iniciando o curso de aprendizagem com os do grupo de aprendizes que já estão inseridos no mercado de trabalho. A média obtida para as respostas dos iniciantes foi de 26,63 e a dos que estão trabalhando, de 25,83. No contraste avaliado pelo teste $\mathrm{t}$, confirma-se que não há diferença significativa entre essas médias $(\mathrm{p}=0,297)$. 
No Gráfico 1B, mostram-se as médias calculadas para os aprendizes com até 16 anos de idade confrontada com a opinião média dos jovens maiores dessa idade. Os valores comparados foram de 26,89 para os primeiros e de 25,76 para os maiores. Nesse caso, também não existiu diferença significativa, sendo o valor da significância do resultado maior que $0,05(\mathrm{p}=0,128)$.

O Gráfico $1 \mathrm{C}$ representa a análise feita entre os respondentes do sexo feminino e os do sexo masculino. As médias para eles foram de 26,84 e 25,27, respectivamente. Quando avaliada a homogeneidade da variância pelo teste de Levene, verificou-se que esta era heterogênea, e, portanto, o teste $t$ se fez com estimativas de variância separadas para os gêneros. Com tal configuração, as médias não podem ser consideradas diferentes, com 5\% de significância. Entretanto, observando o valor-p, pode-se afirmar que, com uma confiança de $90 \%$, elas podem, sim, ser avaliadas como estatisticamente diferentes $(\mathrm{p}=0,080)$.

Gráfico 1 - Variação da imagem em relação ao ensino
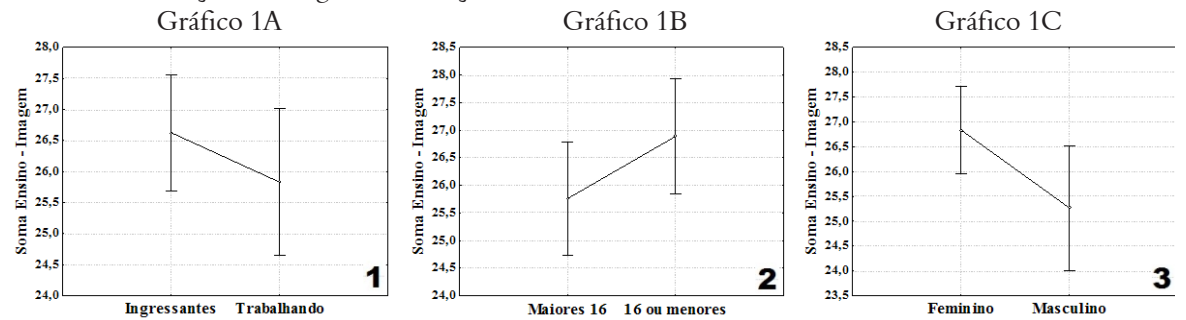

Fonte: os autores.

O Gráfico 2A, expõe a comparação das notas médias para a satisfação com o ensino atribuídas pelo grupo de alunos ingressantes ao curso de aprendizagem e as atribuídas pelo grupo de alunos inseridos no mercado de trabalho. Os valores calculados foram muito próximos, alcançando 26,38 para o primeiro grupo e 25,97 para o segundo. $\mathrm{O}$ teste $\mathrm{t}$ indica que não existe diferença entre elas $(\mathrm{p}=0,580)$.

No Gráfico 2B, quando a condição utilizada para efetuar os contrastes foi a idade, colocada a média da opinião dos aprendizes com idade igual ou inferior a 16 anos em avaliação diante dos maiores dessa idade, também não existiu diferença significativa entre elas. Os valores foram 26,44 e 26 , respectivamente, e o valor-p foi igual a 0,53 . 
Gráfico 2 - Variação de satisfação em relação ao ensino
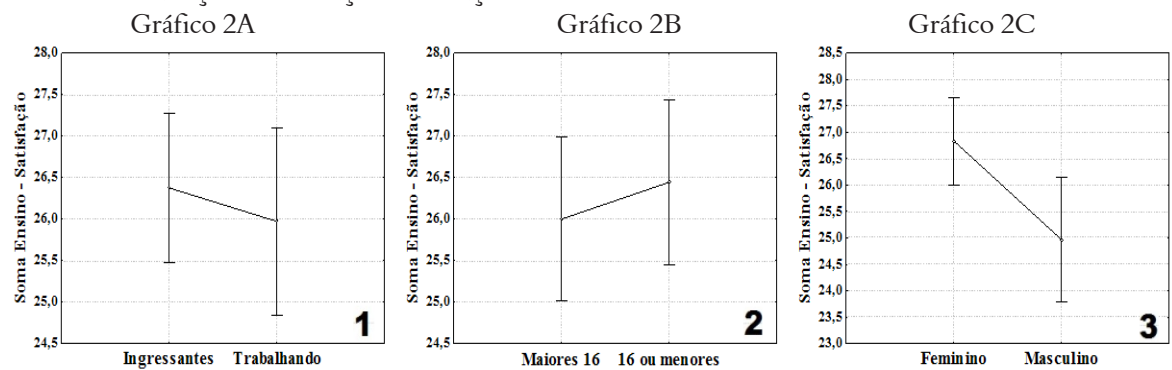

Fonte: os autores.

O Gráfico 2C representa a comparação feita entre as médias dos respondentes do sexo feminino e os do sexo masculino e, da mesma forma como aconteceu em relação à avaliação da imagem, o teste t teve que ser feito com estimativas de variância separadas em razão da falta de homogeneidade desta, segundo o teste de Levene. Após a correção, o teste t mostrou que a média de 26,84, calculada para o gênero feminino, foi significativamente maior $(\mathrm{p}=0,035)$ do que 24,97 , o valor médio para o gênero masculino.

A respeito da instituição que proporciona o curso, os jovens foram questionados acerca da atuação do Instituto Crescer quanto à seleção dos alunos, sua atuação no acompanhamento destes e sua relação com empresas parceiras. Os resultados das análises realizadas são apresentados no Gráfico 3.

Gráfico 3 - Gráficos da variação da imagem com relação ao Instituto Crescer.
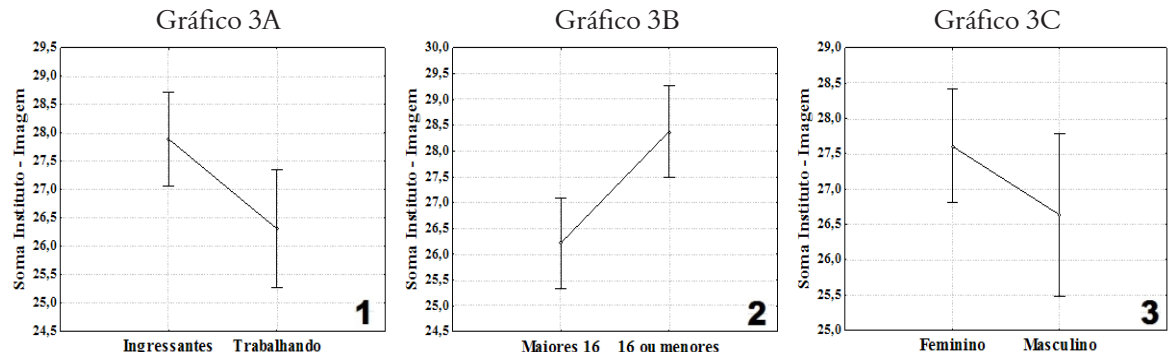

Fonte: os autores.

No Gráfico 3A, exibe-se a comparação das notas médias, no que diz respeito à imagem, atribuídas pelo grupo de alunos que está iniciando o curso de aprendizagem e pelo grupo que já trabalha. A média do primeiro grupo foi de 27,89 e do segundo, 26,31 . No teste $\mathrm{t}$ se comprovou a diferença significativa que há entre 
esses valores $(p=0,020)$, confirmando que a percepção da imagem dos aprendizes que têm ingressado recentemente no programa do Instituto é maior do que a dos mais antigos.

No Gráfico 3B foram colocadas em comparação a opinião dos aprendizes com idade igual ou inferior a 16 anos e a opinião dos maiores dessa idade. Dessa mesma forma que na condição anterior, existiu diferença significativa entre as notas médias, embora nesse caso as estimativas tenham sido feitas com variâncias separadas, pois o teste de Levene foi significante. Os mais jovens tiveram uma média de 28,38 , a qual foi maior ( $\mathrm{p}=0,00$ ) do que 26,22 , o valor da média para os aprendizes maiores.

O Gráfico 3C se representa a comparação feita entre os respondentes do sexo feminino e do masculino. Para essa condição, a imagem em relação ao Instituto não denota diferença significativa entre as médias $(\mathrm{p}=0,170)$. Os valores foram, para o sexo feminino, 27,61, e para o masculino, 26,63.

Na sequência, realizaram-se as comparações das satisfações médias a respeito do Instituto Crescer, considerando as três condições trabalhadas. Os resultados são mostrados no Gráfico 4.

Gráfico 4 - Variação da satisfação em relação ao Instituto Crescer
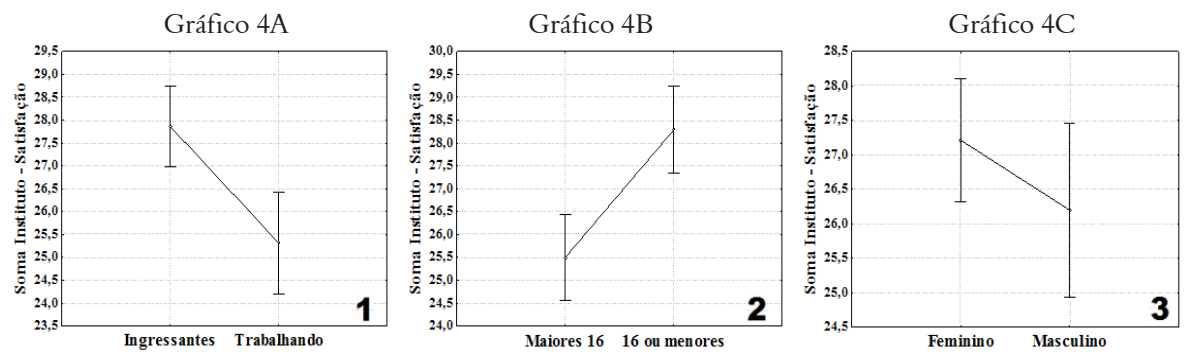

Fonte: os autores.

O Gráfico 4A mostra a comparação das notas médias atribuídas pelo grupo de alunos ingressantes no curso de aprendizagem e as do grupo que já está inserido no mercado de trabalho. A média das respostas dos iniciantes foi de 27,86 e a os que já estão trabalhando, de 25,31. A Falta de homogeneidade da variância, comprovada pelo teste de Levene, fez com que fossem comparadas as médias com estimativas de variância separadas. O valor-p de 0,001 demonstrou que existe diferença significativa entre as médias. 
A comparação entre a opinião dos jovens maiores de 16 anos com a dos aprendizes com idade igual ou inferior é exibida no Gráfico 4B. As médias obtidas para essa comparação foram de 25,50 para o primeiro grupo e de 28,29 para o segundo, representando também uma diferença significativa entre as médias, em que p foi igual a 0,00 . O teste de Levene havia mostrado heterogeneidade da variância e por isso o teste $\mathrm{t}$ foi realizado com estimativas separadas de variância.

No Gráfico 4C, expõe-se a relação entre as notas dos respondentes do sexo feminino e do sexo masculino; o valor-p encontrado foi 0,19 , o qual indica que não existe diferença significativa entre as médias. Assim, o valor de 27,21 para o sexo feminino e de 26,20 para o sexo masculino são estatisticamente iguais.

No que se refere ao desenvolvimento pessoal dos aprendizes, os dados levantados provêm das expectativas que eles manifestam para quatro assuntos: o programa ao ingressar, o programa na atualidade, o crescimento pessoal e suas expectativas de crescimento profissional. Assim, as médias têm valores maiores do que as dos temas antes analisados, que foram avaliados pelas notas atribuídas a três frases.

No Gráfico 5 são apresentados os resultados para as condições definidas para o estudo a respeito da imagem percebida. Assim, no Gráfico 5A é representada a comparação das notas médias referidas ao desenvolvimento pessoal, atribuídas pelo grupo de alunos que está iniciando o curso de aprendizagem e pelo grupo de alunos que está trabalhando. A média obtida das respostas dos iniciantes foi de 37,48 e a dos que já estão trabalhando, de 35,20. O teste t mostra que existe diferença significativa entre as médias $(\mathrm{p}=0,049)$.

Gráfico 5 - Variação da imagem em relação ao desenvolvimento pessoal do jovem
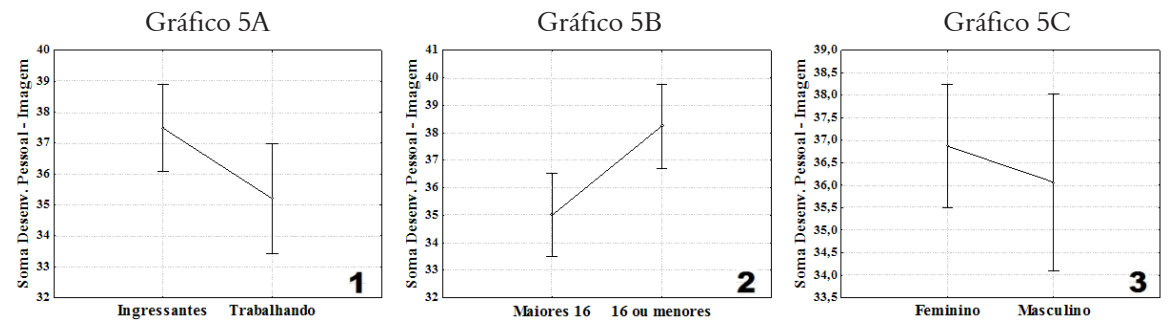

Fonte: os autores.

No Gráfico 5B exibe-se o contraste entre a opinião dos jovens maiores de 16 anos e a dos que têm idade igual ou inferior a ela. Nessa condição também existiu diferença significativa entre as notas médias, que foi de 35 para os maiores e de 38,24 para os menores $(p=0,004)$. O teste $t$ teve que ser feito com estimativas de variância 
separadas para os grupos, uma vez que o resultando do teste de Levene mostrou que as variâncias eram heterogêneas.

Por fim, no Gráfico 5C, está representada a comparação entre os respondentes do sexo feminino e os do sexo masculino. As notas médias nas respostas de ambos os sexos são muito próximas, com 36,87 para o gênero feminino e 36,07 para o masculino. O teste t confirma a igualdade dessas médias, com um valor-p de 0,50.

Quanto à satisfação dos aprendizes com seu desenvolvimento pessoal, os resultados obtidos são expostos no Gráfico 6, que mostra no Gráfico 6A a comparação da média para a condição de alunos ingressantes e dos que trabalham. Os valores calculados foram de 37,34 e 35,71, respectivamente, e o teste t efetuado mostrou que não se pode rejeitar a igualdade $(\mathrm{p}=0,070)$ dessas médias para a significância de $5 \%$ adotada.

Gráfico 6 - Variação da satisfação em relação ao desenvolvimento pessoal do jovem
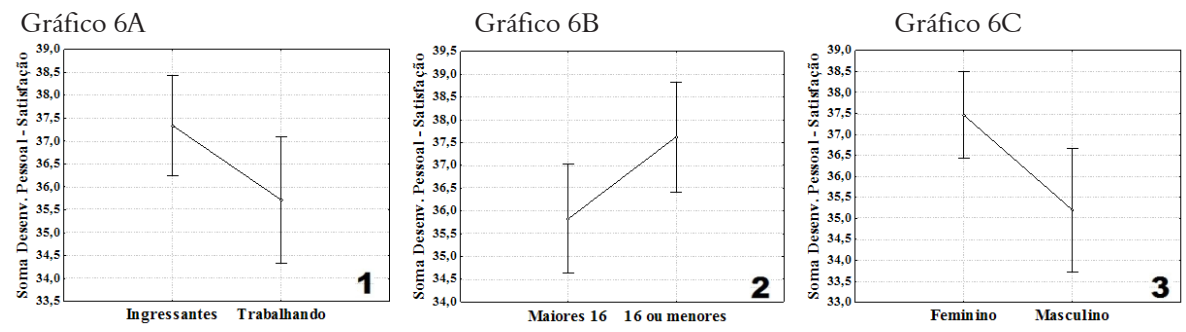

Fonte: os autores.

No Gráfico 6B, a média que se comparou é a calculada para os aprendizes maiores de 16 anos com a obtida para os que têm idade igual ou inferior. Os valores foram de 35,83 e 37,62, respectivamente. Como o resultado do teste de Levene indicou que as variâncias não foram homogêneas, o teste $t$ foi desenvolvido com estimativas de variância separadas. O valor-p obtido foi de 0,039 , o que implica que essas médias são estatisticamente diferentes.

No Gráfico 6C, relativo à comparação entre as médias dos respondentes do sexos feminino e masculino, os valores médios foram de 37,46 e 35,20, respectivamente, para tais categorias. Da mesma forma que no contraste pela condição idade do aprendiz, também para o gênero foi necessário fazer o teste $\mathrm{t}$ com estimativas de variância separadas, uma vez que não existiu homogeneidade na variância para os grupos. O resultado do contraste teve significância $(p=0,036)$, demonstrando que as satisfações médias são diferentes conforme o sexo do aprendiz. 
Os resultados apresentados sobre as comparações de médias de todas as condições estabelecidas são expostos na Tabela 1.

Tabela 1 - Comparações das médias

\begin{tabular}{|c|c|c|c|c|c|c|c|c|c|c|c|c|c|c|c|c|c|}
\hline \multirow{7}{*}{ 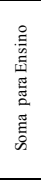 } & \multirow{7}{*}{ 苛 } & & Média & Média & \multirow{2}{*}{$\mathrm{t}$ calc. } & \multirow{2}{*}{$\mathrm{gl}$} & \multirow{2}{*}{ Valor-p } & \multirow{2}{*}{$\begin{array}{l}\mathrm{t} \text { var. } \\
\text { sep. }\end{array}$} & \multirow{2}{*}{ Valor-p } & \multirow{2}{*}{$\begin{array}{c}\mathrm{N}^{\circ} \\
\text { Ingres. }\end{array}$} & \multirow{2}{*}{\begin{tabular}{|c|}
$\mathrm{N}^{\circ}$ \\
Trabalha \\
\end{tabular}} & \multirow{2}{*}{\begin{tabular}{|c|} 
DesvPad \\
Ingres.
\end{tabular}} & \multirow{2}{*}{$\begin{array}{l}\text { DesvPad } \\
\text { Trabalha }\end{array}$} & \multicolumn{2}{|c|}{ Variância } & \multicolumn{2}{|c|}{ Levene } \\
\hline & & Condição & Ingres. & \begin{tabular}{|l|} 
Trabalha \\
\end{tabular} & & & & & & & & & & F calc. & Valor-p & F calc. & Valor-p \\
\hline & & Valores & \begin{tabular}{|l|}
26,62500 \\
\end{tabular} & \begin{tabular}{|l}
25,82857 \\
\end{tabular} & \begin{tabular}{ll|}
1,049 \\
\end{tabular} & \begin{tabular}{|l|}
89 \\
\end{tabular} & \begin{tabular}{|l|}
0,297 \\
\end{tabular} & 1,053 & 0,296 & 56 & 35 & \begin{tabular}{|l|}
3,539710 \\
\end{tabular} & 3,493572 & \begin{tabular}{|l|}
1,027 \\
\end{tabular} & 0,952 & \begin{tabular}{|l|}
0,153 \\
\end{tabular} & \begin{tabular}{|l|}
0,697 \\
\end{tabular} \\
\hline & & Condição & $>16$ & $=$ ou $<16$ & & & & & & $>16$ & $=$ ou $<16$ & $>16$ & $=$ ou $<16$ & & & & \\
\hline & & Valores & \begin{tabular}{|l|}
25,76087 \\
\end{tabular} & \begin{tabular}{|l|}
26,88889 \\
\end{tabular} & $-1,538$ & \begin{tabular}{|l|}
89 \\
\end{tabular} & 0,128 & 1,544 & 0,127 & 46 & 45 & \begin{tabular}{|l|}
4,001026 \\
\end{tabular} & 2,893741 & \begin{tabular}{|l|}
1,912 \\
\end{tabular} & 0,033 & 3,323 & 0,072 \\
\hline & & Condição & Fem. & Masc. & & & & & & Fem. & Masc. & Fem. & Masc. & & & & \\
\hline & & Valores & \begin{tabular}{|l}
26,83607 \\
\end{tabular} & 25,26667 & \begin{tabular}{|l|}
2,031 \\
\end{tabular} & \begin{tabular}{|l|}
89 \\
\end{tabular} & \begin{tabular}{|l|}
0,045 \\
\end{tabular} & 1,792 & 0,080 & 61 & 30 & 2,961871 & 4,322622 & \begin{tabular}{|l|}
2,130 \\
\end{tabular} & 0,014 & 8,369 & \begin{tabular}{|l|l|}
0,005 \\
\end{tabular} \\
\hline \multirow{6}{*}{ 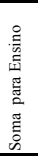 } & \multirow{6}{*}{ 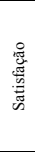 } & Condição & Ingres. & \begin{tabular}{|l|} 
Trabalha \\
\end{tabular} & & & & & & Ingres. & \begin{tabular}{|l|} 
Trabalha \\
\end{tabular} & Ingres. & Trabalha & & & & \\
\hline & & Valores & \begin{tabular}{|l|}
26,37500 \\
\end{tabular} & \begin{tabular}{|l|}
25,97143 \\
\end{tabular} & 0,556 & \begin{tabular}{|l|}
89 \\
\end{tabular} & \begin{tabular}{|l|}
0,580 \\
\end{tabular} & 0,606 & 0,546 & 56 & 35 & \begin{tabular}{|l}
3,783036 \\
\end{tabular} & 2,560856 & \begin{tabular}{|l|}
2,182 \\
\end{tabular} & 0,017 & 2,000 & \begin{tabular}{|l|l|}
0,161 \\
\end{tabular} \\
\hline & & Condição & $>16$ & $=$ ou $<16$ & & & & & & $>16$ & $=$ ou $<16$ & $>16$ & $=\mathrm{ou}<16$ & & & & \\
\hline & & Valor & \begin{tabular}{|l|l|}
26,00000 \\
\end{tabular} & 26,44444 & $-0,630$ & \begin{tabular}{|l|}
89 \\
\end{tabular} & \begin{tabular}{|l|}
0,531 \\
\end{tabular} & $-0,630$ & 0,530 & 46 & 45 & 3,444803 & 3,286028 & \begin{tabular}{|l|}
1,099 \\
\end{tabular} & 0,755 & 0,270 & 0,605 \\
\hline & & Condição & Fem. & Masc. & & & & & & Fem. & Masc. & Fem. & Masc. & & & & \\
\hline & & Valores & \begin{tabular}{|l}
26,83607 \\
\end{tabular} & 24,96667 & \begin{tabular}{|l|l|}
2,575 \\
\end{tabular} & 89 & 0,012 & 2,183 & 0,035 & 61 & 30 & 2,583153 & 4,327007 & \begin{tabular}{|l|}
2,806 \\
\end{tabular} & 0,001 & 7,787 & 0,006 \\
\hline \multirow{6}{*}{ 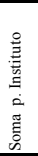 } & \multirow{6}{*}{ 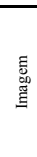 } & Condição & Ingres. & Trabalha & & & & & & Ingres. & Trabalha & Ingres. & Trabalha & & & & \\
\hline & & Valores & \begin{tabular}{|l|}
27,89286 \\
\end{tabular} & \begin{tabular}{|l|}
26,31429 \\
\end{tabular} & \begin{tabular}{|l|l|}
2,367 \\
\end{tabular} & \begin{tabular}{|l|}
89 \\
\end{tabular} & \begin{tabular}{|l|}
0,020 \\
\end{tabular} & 2,327 & 0,023 & 56 & 35 & \begin{tabular}{|l}
3,007134 \\
\end{tabular} & 3,233751 & \begin{tabular}{|l|}
1,156 \\
\end{tabular} & 0,621 & 1,007 & \begin{tabular}{|l|l}
0,318 \\
\end{tabular} \\
\hline & & Condição & $>16$ & $=\mathrm{ou}<16$ & & & & & & $>16$ & $=$ ou $<16$ & $>16$ & $=\mathrm{ou}<16$ & & & & \\
\hline & & Valores & \begin{tabular}{|l|l|}
26,21739 \\
\end{tabular} & \begin{tabular}{|l|l}
28,37778 \\
\end{tabular} & \begin{tabular}{|l|l}
$-3,436$ \\
\end{tabular} & \begin{tabular}{|l|}
89 \\
\end{tabular} & \begin{tabular}{|l|}
0,001 \\
\end{tabular} & $-3,456$ & 0,001 & 46 & 45 & \begin{tabular}{|l|l}
3,693316 \\
\end{tabular} & 2,059224 & \begin{tabular}{|l|}
3,217 \\
\end{tabular} & 0,000 & 5,452 & \begin{tabular}{|l|}
0,022 \\
\end{tabular} \\
\hline & & Condição & Fem. & Masc. & & & & & & Fem. & Masc. & Fem. & Masc. & & & & \\
\hline & & Valores & \begin{tabular}{|l}
27,60656 \\
\end{tabular} & 26,63333 & \begin{tabular}{|l|}
1,382 \\
\end{tabular} & \begin{tabular}{|l|}
89 \\
\end{tabular} & \begin{tabular}{|l|l|}
0,170 \\
\end{tabular} & 1,243 & 0,220 & 61 & 30 & \begin{tabular}{|l}
2,782557 \\
\end{tabular} & 3,819039 & \begin{tabular}{|l|}
1,884 \\
\end{tabular} & 0,039 & 1,456 & 0,231 \\
\hline \multirow{6}{*}{ 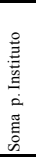 } & \multirow{6}{*}{ 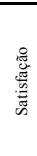 } & Condição & Ingres. & \begin{tabular}{|l|} 
Trabalha \\
\end{tabular} & & & & & & Ingres. & \begin{tabular}{|l|} 
Trabalha \\
\end{tabular} & Ingres. & Trabalha & & & & \\
\hline & & Valores & \begin{tabular}{|l|l}
27,85714 \\
\end{tabular} & \begin{tabular}{|l|}
25,31429 \\
\end{tabular} & $\begin{array}{l}3,581 \\
\end{array}$ & \begin{tabular}{|l|}
89 \\
\end{tabular} & \begin{tabular}{|l|}
0,001 \\
\end{tabular} & 3,398 & 0,001 & 56 & 35 & \begin{tabular}{|l|l}
2,981327 \\
\end{tabular} & 3,747716 & \begin{tabular}{ll|}
1,580 \\
\end{tabular} & 0,129 & 4,030 & 0,048 \\
\hline & & Condição & $>16$ & $=$ ou $<16$ & & & & & & $>16$ & $=$ ou $<16$ & $>16$ & $=$ ou $<16$ & & & & \\
\hline & & Valores & 25,50000 & \begin{tabular}{|l|}
28,28889 \\
\end{tabular} & $-4,118$ & \begin{tabular}{|l|}
89 \\
\end{tabular} & \begin{tabular}{|l|}
0,000 \\
\end{tabular} & $-4,147$ & 0,000 & 46 & 45 & 4,135215 & 1,902417 & \begin{tabular}{|l|}
4,725 \\
\end{tabular} & 0,000 & \begin{tabular}{|l|}
11,355 \\
\end{tabular} & 0,001 \\
\hline & & Condição & Fem. & Masc. & & & & & & Fem. & Masc. & Fem. & Masc. & & & & \\
\hline & & Valores & \begin{tabular}{|l|l|}
27,21311 \\
\end{tabular} & 26,20000 & 1,301 & \begin{tabular}{|l|}
89 \\
\end{tabular} & 0,197 & 1,231 & 0,224 & 61 & 30 & \begin{tabular}{|l|}
3,291984 \\
\end{tabular} & 3,872093 & 1,383 & 0,288 & 0,280 & $\begin{array}{l}0,598 \\
\end{array}$ \\
\hline \multirow{6}{*}{ 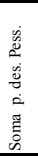 } & \multirow{6}{*}{ 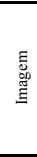 } & \begin{tabular}{|l} 
Condição \\
\end{tabular} & I & $\mathrm{T}$ & & & & & & 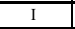 & $\mathrm{T}$ & I & $\bar{T}$ & & & & \\
\hline & & Valores & \begin{tabular}{|l|l}
37,48214 \\
\end{tabular} & 35,20000 & \begin{tabular}{|l|}
1,999 \\
\end{tabular} & \begin{tabular}{|l|}
89 \\
\end{tabular} & \begin{tabular}{|l|}
0,049 \\
\end{tabular} & 1,801 & 0,078 & 56 & 35 & 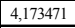 & 6,733586 & \begin{tabular}{|l|}
2,603 \\
\end{tabular} & 0,002 & \begin{tabular}{|l|}
3,558 \\
\end{tabular} & 0,063 \\
\hline & & Condição & $>16$ & $=$ ou $<16$ & & & & & & $>16$ & $=$ ou $<16$ & $>16$ & $=$ ou $<16$ & & & & \\
\hline & & Valores & \begin{tabular}{|l|l}
35,00000 \\
\end{tabular} & \begin{tabular}{|l|}
38,24444 \\
\end{tabular} & \begin{tabular}{|l|l|}
$-2,997$ \\
\end{tabular} & \begin{tabular}{|l|}
89 \\
\end{tabular} & \begin{tabular}{|l|}
0,004 \\
\end{tabular} & $-3,025$ & 0,004 & 46 & 45 & \begin{tabular}{|c|}
6,931410 \\
\end{tabular} & 2,186275 & 10,052 & 0,000 & 14,560 & 0,000 \\
\hline & & Condição & Fem. & Masc. & & & & & & Fem. & Masc. & Fem. & Masc. & & & & \\
\hline & & Valores & $36,8688$. & 36,06667 & 0,666 & 89 & 0,507 & 0,675 & 0,502 & 6 & 30 & 5,472584 & 5,258381 & \begin{tabular}{|l|}
1,083 \\
\end{tabular} & 0,834 & 0,117 & 0,733 \\
\hline & & \begin{tabular}{|l} 
Condição \\
\end{tabular} & $\mathrm{I}$ & $\mathrm{T}$ & & & & & & I & $\mathrm{T}$ & $\mathrm{I}$ & $\mathrm{T}$ & & & & \\
\hline 孛 & & Valores & \begin{tabular}{|l|l}
37,33929 \\
\end{tabular} & 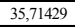 & $\begin{array}{ll}1,835 \\
\end{array}$ & \begin{tabular}{|l|}
89 \\
\end{tabular} & \begin{tabular}{|l|}
0,070 \\
\end{tabular} & 1,788 & 0,078 & 56 & 35 & \begin{tabular}{|l|l|}
3,927881 \\
\end{tabular} & 4,389636 & \begin{tabular}{|l|}
1,249 \\
\end{tabular} & 0,455 & \begin{tabular}{|l|}
2,340 \\
\end{tabular} & 0,130 \\
\hline 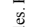 & 䒻 & Condição & $>16$ & $=$ ou $<16$ & & & & & & $>16$ & $=$ ou $<16$ & $>16$ & $=$ ou $<16$ & & & & \\
\hline 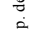 & & Valores & \begin{tabular}{|l|}
35,82609 \\
\end{tabular} & 37,62222 & $-2,096$ & \begin{tabular}{|l|}
89 \\
\end{tabular} & \begin{tabular}{|l|}
0,039 \\
\end{tabular} & $-2,105$ & 0,039 & 46 & 45 & \begin{tabular}{|l|l|}
4,808808 \\
\end{tabular} & 3,185827 & \begin{tabular}{|l|}
2,278 \\
\end{tabular} & 0,007 & 4,705 & 0,033 \\
\hline 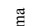 & & Condição & Fem. & Masc. & & & & & & Fem. & Masc. & $\mathrm{Fe}$ & Masc. & & & & \\
\hline & & Valores & \begin{tabular}{|l|}
37,45902 \\
\end{tabular} & 35,20000 & 2,503 & \begin{tabular}{|l|}
89 \\
\end{tabular} & \begin{tabular}{|l|}
0,014 \\
\end{tabular} & 2,166 & 0,036 & 61 & 30 & 3,344517 & 5,208746 & 2,425 & 0,004 & 10,113 & 0,002 \\
\hline
\end{tabular}

Fonte: os autores.

\section{CONSIDERAÇÕES FINAIS}

O objetivo proposto de investigar a percepção de imagem e satisfação dos aprendizes em relação ao ensino, a instituição que abriga o programa de aprendizagem e seu desenvolvimento pessoal foi alcançado pela análise dos dados obtidos com o questionário aplicado. A caracterização dos participantes do programa, realizada a partir da parte inicial do instrumento de coleta de informações, confirma a função social do programa de aprendizagem.

Constatou-se que a maioria dos jovens aprendizes considera ser significativo iniciar sua vida profissional como aprendizes, pois essa experiência auxiliará sua inserção no mercado de trabalho, de modo que também contribui para definir a 
carreira profissional que deseja seguir, além disso, influencia a cursar ensino superior, visando sempre aprimorar seus conhecimentos profissionais.

Ao se considerar os valores mínimos e máximos que as percepções sobre a imagem e a satisfação podem atingir, conforme o aspecto que se considere, percebese que os valores foram altos. Nas médias da imagem sobre o ensino, o valor mínimo foi de 25,26 e para a satisfação, de 24,96. Para o Instituto, os mínimos foram de 26,21 e 25,5 para a imagem e satisfação, respectivamente. Quanto à expectativa de desenvolvimento pessoal, a imagem teve uma média mínima de 35 enquanto o valor para a satisfação foi de 35,20. Assim, os resultados apontaram que em todas as categorias as médias foram maiores que $80 \%$ do valor máximo possível tanto para a imagem quanto para a satisfação, seja para os aprendizes iniciantes seja para os mais antigos. Confirma-se, portanto, que as respostas dos aprendizes são muito favoráveis ao desenvolvimento do programa.

Nas análises mais detalhadas, quando contrastadas as condições definidas para a análise, alguns padrões puderam ser reconhecidos. Excluindo os casos em que não pode ser descartada a igualdade das médias, os aprendizes ingressantes atribuem maiores pontuações para a imagem e satisfação. Do mesmo modo, verificam-se médias mais elevadas para os aprendizes de menor idade quando comparadas às dos maiores. E as mulheres têm valores médios mais altos que os homens.

Algumas limitações deste artigo podem ser mencionadas, entre elas o fato de se tratar apenas das expectativas dos aprendizes matriculados em uma única instituição, o Instituto Crescer. Outro aspecto limitante é não terem sido avaliados os jovens que, tendo feito um contato de aprendizagem, já foram absorbidos pelas empresas, isto é, que foram efetivados nos cargos. Assim, propõe-se, para pesquisas futuras, continuar os estudos sobre a aprendizagem, considerando os aspectos não abordados. Por outra parte, sugere-se que também sejam trabalhados os aspectos legais e contábeis da contratação dos aprendizes.

\section{REFERÊNCIAS}

ANDRADE, M. M. Introdução à metodologia do trabalho científico. 4. ed. São Paulo: Atlas, 1999.

BABBIE, E. Métodos de pesquisa de survey. Belo Horizonte: UFMG, 2003. 
BRASIL. Decreto-lei n. 5.452, de 01 de maio de 1943. Aprova a Consolidação das Leis do Trabalho. Diário Oficial da União, Brasília, DF, 03 maio 1943. Disponível em: < http://www2.camara.leg.br/legin/fed/declei/1940-1949/decretolei-5452-1-maio-1943-415500-publicacaooriginal-1-pe.html>. Acesso em: 17 set. 2013.

BRASIL. Decreto-lei n. 5.598, de 01 de dezembro de 2005. Regulamenta a contratação de aprendizes e dá outras providências. Diário Oficial da União, Brasília, DF, 03 dez. 2005a. Disponível em: < http://www.planalto.gov.br/ ccivil_03/_ato20042006/2005/decreto/d5598.htm > . Acesso em: 17 set. 2013.

BRASIL. Instrução Normativa SIT n. 26, de 20 de dezembro de 2001. Baixa instruções para orientar a fiscalização das condições de trabalho no âmbito dos programas de aprendizagem, 2001. Diário Oficial da União, Brasília, DF, 22 dez. 2001. Disponível em: < http://portal.mte.gov.br/legislacao/instrucao-normativa-n26-de-20-12-2001.htm>. Acesso em: 20 set. 2013.

BRASIL. Lei n. 10.097, de 19 de dezembro de 2000. Altera dispositivos da Consolidação das Leis do Trabalho - CLT, aprovada pelo Decreto-Lei n. 5.452, de 01 de maio de 1943. Diário Oficial da União, Brasília, DF, 21 dez. 2000. Disponível em: < http://www.planalto.gov.br/ccivil_03/LEIS/L10097.htm>. Acesso em: 20 set. 2013.

BRASIL. Lei n. 11.180, de 23 de setembro de 2005. Institui o Projeto Escola de Fábrica, autoriza a concessão de bolsas de permanência a estudantes beneficiários do Programa Universidade para Todos - PROUNI, institui o Programa de Educação Tutorial - PET, altera a Lei n. 5.537, de 21 de novembro de 1968, e a Consolidação das Leis do Trabalho - CLT, aprovada pelo Decreto-Lei n. 5.452, de 01 de maio de 1943, e dá outras providências. Diário Oficial da União, Brasília, DF, 27 dez. 2005b. Disponível em: < http://www.planalto.gov.br/ccivil_03/_ ato2004-2006/2005/lei/L11180.htm>. Acesso em: 17 set. 2013.

BROWN, D. K.; STERN, R. M.; DEARDORFF, A. V. The determinants of child labor: theory and evidence. In: RESEARCH SEMINAR IN INTERNATIONAL ECONOMICS, 2002, Michigan. Annals... The University of Michigan, Sept. 2002.

CÂMARA, S. G.; SARRIERA, J. C. Critério de seleção para o trabalho de adolescentes - jovens: perspectiva dos empregadores. Psicologia em Estudo, v. 6, n. 1, p. 77-84, 2001.

CERVO, A. L.; BERVIAN, P. A. Metodologia científica: para uso dos estudantes universitários. 5. ed. São Paulo: Prentice Hall, 2003. 
CHURCHILL JUNIOR, G. A.; PETER, P. J. Marketing criando valor para os clientes. 2. ed. São Paulo: Saraiva, 2003.

CRISPIM, K. S.; GODOY, J. H. A. Juventude e a questão social no contexto das políticas públicas de trabalho e emprego. Revista Eletrônica de Ciências Sociais, n. 9, p. 134-149, 2010.

DE TONI, D. Administração da imagem de produtos: desenvolvendo um instrumento para a configuração da imagem de produto. 2005. Tese (Doutorado em Administração)-Universidade Federal do Rio Grande do Sul, Porto Alegre, 2005.

DE TONI, D.; MILAN, G. S.; SCHULER, M. Configuração de imagens de serviços: um estudo aplicado aos serviços de fisioterapia disponibilizados por um plano de saúde. In: ENCONTRO ANUAL DA ANPAD, 24., 2005, Brasília. Anais... Campinas: ANPAD, 2005.

EMERSON, P; SOUZA, A. P. Is there a child labor trap? Inter-generational persistence of child labor in Brazil. Economic Development and Cultural Change, v. 51, i. 2, p. 375-398, 2003.

ERSADO, L. Child Labor and Schooling Decisions in Urban and Rural Areas: Cross-Country Evidence. In: AMERICAN AGRICULTURAL ECONOMICS ASSOCIATION ANNUAL MEETING, July 2003, Montreal. Annals... Montreal, 2003. Disponível em:<http://ageconsearch.umn.edu/bitstream/21924/1/ sp03er01.pdf>. Acesso em: 17 set. 2013.

FAN, C. S. Relative wage, child labor, and human capital. Oxford Economic Papers, n. 56, p. 687-700, 2004.

FERRAZ, R. Ensino médio e preparação da juventude para o mercado de trabalho: contradições. 2006. Dissertação (Mestrado em Educação: História, Política e Sociedade)-Pontifícia Universidade Católica de São Paulo, São Paulo, 2006.

FREITAS, M. V.; PAPPA, F. C. Políticas públicas: juventude em pauta. São Paulo: Cortez, 2008.

FRIGOTTO, G. A dupla face do trabalho: criação e destruição da vida. In: FRIGOTTO, G.; CiAVATTA, M. (Org.). A experiência do trabalho e a educação básica. 3. ed. São Paulo: Lamparina, 2010. 
JOSVIAK, M. Políticas públicas e aprendizagem: a participação do Ministério Público do Trabalho na construção de políticas públicas para profissionalizar jovens no Paraná. In: JOSVIAK, M.; BLEY, R. B (Org.). Ser aprendiz! Aprendizagem profissional e políticas públicas: aspectos jurídicos, teóricos e práticos. São Paulo: LTr, 2009.

KASSOUF, A. L.; MCKEE, M.; MOSSIALOS, E. Early entrance to the job market and its effect on adult health: evidence from Brazil. Health Policy and Planning, v. 16, i. 1, p. 21-28, 2001.

KOTLER, P. Administração de marketing. 5. ed. São Paulo: Atlas, 1998.

MACEDO, O. J. V. O sentido da formação para o trabalho e as expectativas em relação ao futuro por parte dos adolescentes aprendizes. 2006. Dissertação (Mestrado em Psicologia)-Universidade Federal da Paraíba, João Pessoa, 2006.

MARTINS, G. A.; THEÓPHILO, C. R. Metodologia da investigação científica para ciências sociais aplicadas. 2. ed. São Paulo: Atlas, 2009.

McKENNA, R. Marketing de relacionamento: estratégias bem-sucedidas para a era do cliente. 15. ed. São Paulo: Campus, 1992.

MINISTÉRIO DO TRABALHO E EMPREGO. Manual da aprendizagem. O que é preciso saber para contratar o jovem aprendiz. Brasília: SPPE, 2009.

NEVES, A. B; RAMOS, C. F. A imagem das Instituições de Ensino Superior e a qualidade do ensino de graduação: a percepção dos acadêmicos do curso de administração. Revista de Economia e Administração, v. 1, n. 1, p. 75-84, 2002.

OLIVEIRA, A. B. Protagonismo juvenil: o programa aprendiz Comgás no município de Campinas. 2009. Dissertação (Mestrado em Educação)-Pontifícia Universidade Católica de São Paulo, Campinas, 2009.

SAMPAIO, R. Propaganda de A a Z: como usar a propaganda para construir marcas e empresas de sucesso. Rio de Janeiro: Campus, 1999.

SANTOS, C. F. Contrato de emprego do adolescente aprendiz. Curitiba: Juruá, 2003.

SCHULER, M.; DE TONI, D. Imagem de produto e comportamento do consumidor: explorando o processo de formação de imagens. In: ENCONTRO ANUAL DA ANPAD, 28., 2004, Curitiba. Anais... Curitiba: ANPAD, 2004. 
SOARES, L. S. Menor aprendiz: obrigação de contratação limites legais. Revista IOB, IOB Trabalhista e Previdenciária, v. 17, n. 214, p. 16-19, 2007.

\section{COMO CITAR ESTE ARTIGO:}

LIZOTE, Suzete Antonieta et al. Programa de aprendizagem: imagem e satisfação — na visão dos aprendizes. RACE, Revista de Administração, Contabilidade Z e Economia, Joaçaba, Ed. Unoesc, v. 14, n. 3, p. 1119-1144, set./dez. 2015.

\& Disponível em: < http://editora.unoesc.edu.br/index.php/race>. Acesso em: dia/ mês/ano.

Lizote, S. A., Verdinelli, M. A., Laurentino, E. C., Santos, L. da S., \& Petrelli, T. C.

$\overleftarrow{1}$ M. (2015). Programa de aprendizagem: imagem e satisfação na visão dos aprendizes.

\& RACE, Revista de Administração, Contabilidade e Economia, 14(3), 1119-1144.

Recuperado em: dia/mês/ano, de http://editora.unoesc.edu.br/index.php/race. 
